\title{
On a Constant in the Theory of Trigonometric Series
}

\section{By Robert F. Church}

The note "A constant in the theory of trigonometric series" in the October 1964 issue of Mathematics of Computation provided us with a test for our recently constructed algorithms for the computation of roots of functions, and for numerical quadrature in the presence of singularities. The latter algorithm, utilizing the Gaussian 8-point quadrature formula applied to sub-intervals of variable length, involves a sufficiently small number of ordinates that computational labor and round-off error do not become problems. Use of these algorithms indicated the value $\alpha_{0}=.3084438$, for the root of the equation $\int_{0}^{3 \times / 2} u^{-\alpha} \cos u d u=0$, differing from the reported value, .30483 , in the third place. To check this result, we made the transformation $u=x^{4}$ to weaken the character of the singularity at the origin, and obtained the following table by conventional numerical quadrature, confirming our result:

$\begin{array}{cc}\alpha & F(\alpha) \\ .308441 & -.99\left(10^{-5}\right) \\ .308442 & -.63\left(10^{-5}\right) \\ .308443 & -.28\left(10^{-5}\right) \\ .308444 & .08\left(10^{-5}\right) \\ .308445 & .44\left(10^{-5}\right) \\ .308446 & .79\left(10^{-5}\right) .\end{array}$

Sperry Rand Research Center

North Road

Sudbury, Massachusetts

Received October 28, 1964.

\section{On a Constant in the Theory of Trigonometric Series}

\section{By Yudell L. Luke, Wyman Fair, Geraldine Coombs and Rosemary Moran}

In a recent note, Boas and Klema [1] considered

$$
F(\alpha)=\int_{0}^{3 \pi / 2} u^{-\alpha} \cos u d u, \quad R(\alpha)<1
$$

and gave some computations from which they concluded that a zero $\alpha_{0}$ of $F(\alpha)$ lies between 0.30483 and 0.30484 . Since their tabulated values of $F(\alpha)$ in the vicinity of the root are given to $8 \mathrm{D}$ and there are eight such entries, it would seem, since $F(\alpha)$ is analytic for $R(\alpha)<1$, that the zero could be given to more places by differencing and making use of ordinary inverse interpolation techniques. It is found

Received December 15, 1964. 